\title{
聚溴化炔丙基喹啉及聚溴化炔丙基叫啶的简便高效制备 及其结构与性质表征
}

\author{
周常明 $a, b$ 陈道勇*, $a, b$ \\ ( ${ }^{a}$ 复旦大学高分子科学系 上海 200433) \\ ( ${ }^{b}$ 聚合物分子工程国家重点实验室 上海 200433)
}

\begin{abstract}
摘要 在本研究工作中, 我们发展了通过澳丙炔(propargyl bromide)分别与喹啉(quinoline)、呋啶(acridine)直接反应来制 备聚溴化炔丙基喹啉[poly(propargyl quinolinium bromide), PPQB]和聚埧化炔丙基呋啶[poly(propargyl acridinium bromide), PPAB]的新方法. PPQB 与 PPAB 的主链均为聚乙炔结构, 侧链分别为季铵化喹啉盐和呋啶盐基团, 是一类单取 代的聚电解质型聚乙炔(polyelectrolyte polyacetylenes, PE-PAs). 我们提出了上述反应的机理, 即溴丙炔与喹啉或呋啶 反应生成季铵盐产物并导致产物结构中炔基基团的活化, 使得季铵盐发生自聚合反应生成 PPQB 或 PPAB. PPQB 及 PPAB 具有较高的分子量(PPQB 及 PPAB 的分子量分别为 $1.8 \times 10^{5}$ 和 $\left.8.1 \times 10^{4} \mathrm{~g} / \mathrm{mol}\right)$. 进一步的表征表明, PPQB 和 PPAB 均具有良好的苂光活性和导电性能.
\end{abstract}

关键词 聚电解质; 单取代聚乙炔; 无催化剂; 苂光; 导电性

\section{Facile and Efficient Catalyst-Free Preparation of Poly(propargyl quinolinium bromide) and Poly(propargyl acridinium bromide) and Characterizations of Their Structures and Properties}

\author{
Zhou, Changming ${ }^{a, b} \quad$ Chen, Daoyong*,a,b \\ ( ${ }^{a}$ Department of Macromolecular Science, Fudan University, Shanghai 200433) \\ ( ${ }^{b}$ State Key Laboratory of Molecular Engineering of Polymer, Shanghai 200433)
}

\begin{abstract}
In this paper, we report facile and efficient preparation of poly(propargyl quinolinium bromide) (PPQB) and poly(propargyl acridinium bromide) (PPAB). Both PPQB and PPAB are conjugated polymers with polyacetylene as backbones. Besides, they are polyelectrolytes since the side groups of PPQB and PPAB are quaternized quinolinium and acridinium, respectively. Therefore, PPQB and PPAB are conjugated polyelectrolytes, which are promising in many important applications. PPQB was prepared by reaction of the mixture of propargyl bromide (PB) and quinoline in DMF at $60{ }^{\circ} \mathrm{C}$ for 100 h. PPAB was synthesized under the same conditions via the same process except that acridine was used in the place of quinoline. The preparations are very simple and efficient, which need no any catalyst or initiator. The as-prepared PPQB and PPAB were characterized by elemental analysis and GPC-MALLS. Elemental analysis confirmed that PPQB and PPAB were the respective homopolymers of propargyl quinolinium bromide (PQB) and propargyl acridinium bromide (PAB). The molecular weights of the PPQB and PPAB were measured to be $1.8 \times 10^{5}$ and $8.1 \times 10^{4} \mathrm{~g} / \mathrm{mol}$, respectively, indicating efficient polymerizations of the respective monomers $\mathrm{PQB}$ and $\mathrm{PAB}$. A tentative mechanism for the polymerizations was proposed as follows: firstly, the tertiary amine group of quinoline or acridine was quaternized by PB, leading to activation of the carbon-carbon triple bonds of the resultant quaternized salt; then, the nucleophilic attack on the triple bonds by pyridine resulted in carbon anions, which initiated the anionic polymerization of the quaternized salt. Furthermore, fluorescent and conductive properties of PPQB and PPAB were studied. Fluorescent measurements revealed that both PPQB and PPAB solutions had relatively strong fluorescent emissions, and the conductivity measurements demonstrated good conductivities of PPQB and PPAB films. Compared with poly(propargyl pyridinium bromide) that we reported previously, PPQB and PPAB are much less hygroscopic when exposed to air, so that PPQB and PPAB films are stable in the air. This broadens the applications of the two polymers.

Keywords polyelectrolyte; mono-substituted polyacetylene; catalyst-free; fluorescence; conductivity
\end{abstract}

\footnotetext{
*E-mail: chendy@fudan.edu.cn; Tel.: +86-21-65643989

Received November 16, 2013; published November 28, 2013.

Supporting information for this article is available free of charge via the Internet at http://sioc-journal.cn.

Project supported by the National Natural Science Foundation of China (Nos. 91127030 and 21334001) and the Ministry of Science and Technology of China (No. 2011CB932503).

项目受国家自然科学基金(Nos. 91127030,21334001)及科技部 973 项目基金(No. 2011CB932503)资助.
} 


\section{1 引言}

共轭聚合物(conjugated polymer)是主链具有电子离 域结构的一类聚合物, 近年来被广泛地应用在发光二极 管、太阳能电池、生物传感器、电致变色元件, 有机电 子器件和非线性光学元件等领域 ${ }^{[1 \sim 10]}$. 其中, 聚乙炔 (polyacetylene, PA) 是结构最为简单且最早被合成的共 轭聚合物; 20 世纪 70 年代, 人们发现经过掺杂的 PA 薄 膜具有良好的导电性能, 从而使得 PA 的研究成为热点 领域 ${ }^{[1116]}$. 但是 PA 本身的性质缺陷对其商业化应用造 成影响: 其溶解性差、难以熔融的特点使得难以对 PA 材料进行成型加工, 且材料的稳定性也因 PA 在空气中 易被氧化而变差 ${ }^{[17,18]}$. 为解决这些问题, 人们开始研究 取代聚乙炔(substituted polyacetylenes) ${ }^{[17 \sim 24]}$. 其中结构 单元的侧链带有离子化基团的取代聚乙炔被称为聚电 解质型聚乙炔(polyelectrolyte polyacetylenes, PE-PAs). PE-PAs 在水中有着良好的溶解性, 可以通过对环境友 好的方法(environmental benign methods)进行加工和处 理; 此外, PE-PAs 还具有与生物活性分子相互作用的能 力, 使得 PE-PAs 在生物化学领域有着广泛的应用前景.

目前, 制备 PE-PAs 的方法主要有: 对聚乙炔型聚 合物进行后处理修饰 ${ }^{[25]}$, 以及对取代乙炔类的单体直 接进行聚合反应 ${ }^{[26 ~ 29]}$. 这些合成方法需要使用催化剂 或助剂; 这些催化剂或助剂对外部空气或水汽敏感, 反 应多需要在无水无氧条件下进行, 因而反应条件较苛 刻; 由于催化剂或助剂会在产物中残留, 会对产物性能 造成影响. 因此发展出无需催化剂或助剂, 且能够在温 和实验条件下合成 PE-PAs 的方法就十分有必要. 在 20 世纪 70 年代, Kabanov 等 ${ }^{[30]}$ 曾经报道过一种无需催化剂 合成 PE-PAs 的方法: 炔丙基衍生物 (propargyl derivatives)如氯丙炔、溴丙炔等能够直接与部分含氮化合物反 应生成 PE-PAs. 但是, 该反应仅能得到齐聚物 ${ }^{[1]} .1991$ 年以后, Blumstein 研究组以及 $\mathrm{Gal}$ 研究组 ${ }^{[31 \sim 35]}$ 报道了通 过 2-炔基吡啶或 4-炔基吡啶与卤代烃反应可以生成 PEPAs. 该方法无需催化剂或其它助剂, 但需要使用炔基 吡啶为原料. 由于炔基吡啶的合成较为困难, 炔基吡啶 的使用增加了该方法的难度, 也限制了所能获得的 PE-PAs 的种类.

在之前的研究工作中, 我们报道了将吡啶(pyridine, PD)与溴丙炔 (propargyl bromide, PB) 在 DMF 溶剂中直 接混合后反应, 可以得到一种 PE-PAs 型聚合物——聚 澳化炔丙基吡啶 [poly(propargyl pyridinium bromide), $\mathrm{PPPB}]^{[36]}$. 研究表明, 该聚合物具有较高的分子量, 较 好的水溶性以及优良的苂光活性. 但是, 一旦暴露在空 气中, PPPB 由于吸水性太强, 会大量吸收空气中的水 分, 从而限制了其应用. 在本研究工作中, 我们进一步 研究了 PB 与其它含有吡啶环结构的吡啶衍生物之间的 反应。研究表明, 利用喹啉 (quinoline, QL) 和呋啶 (acridine, AD) 分别与 PB 反应可获得聚溴化炔丙基喹啉 [poly(propargyl quinolinium bromide), PPQB]及聚溴化炔 丙基吅啶 [poly(propargyl acridinium bromide), PPAB]. 制得的 $\mathrm{PPQB}$ 与 $\mathrm{PPAB}$ 均具有较高的分子量 $\left(\approx 10^{5}\right.$ $\mathrm{g} / \mathrm{mol})$, 说明了这一简单高效的 PE-PAs 合成方法具有 普适性. 进一步的研究表明, PPQB 和 PPAB 均具有良好 的苂光活性. 另外, 与 PPPB 相比, PPQB 及 PPAB 的吸 水性大大降低，因而可以制成在空气中稳定的薄膜用于 聚合物导电性能的测量; 导电性测量结果表明, PPQB 和 PPAB 薄膜均具有较好的导电性. 值得说明的是, PPQB 和 PPAB 的合成及性能研究在文献中均未见报道.

\section{2 结果与讨论}

\section{$2.1 \mathrm{~PB} / \mathrm{QL}$ 及 $\mathrm{PB} / \mathrm{AD}$ 反应产物的分子量及化学结构}

将 $\mathrm{PB} / \mathrm{QL}$ 或者 $\mathrm{PB} / \mathrm{AD}$ 在 $\mathrm{DMF}$ 中反应后得到的产 物进行多次纯化然后干燥(实验部分 4.1 和 4.2 节), 可分 别得到红褐色或浅黄色的固体. 将产物分别溶解在 $\mathrm{DMF}$ 中制成溶液 $(5.0 \mathrm{mg} / \mathrm{mL}$ ), 然后用以 $\mathrm{DMF}$ (含 2.0 $\mathrm{mg} / \mathrm{mL} \mathrm{LiBr}$ ) 为流动相的 GPC-MALLS 进行测试. 各产 物在 GPC 曲线上的样品峰均为一单峰(S2, Supporting Information), 通过 MALLS 可以准确测定各产物的重均 分子量 $\left(M_{\mathrm{w}}\right)$ (由于季铵盐对色谱柱有吸附, 以流出时间 作为依据测定的分子量误差较大). 测试得到的 $\mathrm{PB} / \mathrm{QL}$ 反应产物的分子量为 $M_{\mathrm{w}}=1.8 \times 10^{5}$, 而 $\mathrm{PB} / \mathrm{AD}$ 反应产 物的分子量为 $M_{\mathrm{w}}=8.1 \times 10^{4}$, 这说明上述两个反应体系 中, 均生成了高分子量的聚合产物.

理论上, PB/QL 反应生成的聚合产物有以下 3 种可 能结构: (1) PB 的均聚物; (2) PB 与溴化炔丙基哇啉 (PB 与 $\mathrm{QL}$ 间季铵化反应的产物)的共聚物; (3)溴化炔丙基喹 啉(propargyl quinolinium bromide, PQB)的均聚物 PPQB (Scheme 1). 由于不同结构中各元素的含量存在差异, 可以通过对反应产物进行元素分析(EA)测试来确定其 化学结构. EA 测试结果显示, $\mathrm{PB} / \mathrm{QL}$ 反应产物中各元素 的质量分数分别为: (C $58.35 \%, \mathrm{~N} 5.61 \%, \mathrm{H} 4.15 \%)$. 对 上述 3 种结构的元素含量进行计算, 只有第 3 种结构 (即 PPQB)的计算结果(C $58.13 \%, \mathrm{~N} 5.65 \%, \mathrm{H}$ 4.07\%)最符合 实际测得的该产物各元素含量. 同样, 对于 $\mathrm{PB} / \mathrm{AD}$ 反应 的产物, 其 EA 测试结果(C $64.90 \%, \mathrm{~N} 4.82 \%, \mathrm{H} 4.56 \%$ ) 也显示其与第 3 种结构 [溴化炔丙基叮啶(propargyl acridinium bromide, PAB) 的均聚物 PPAB] 的各元素理论含 量(C $64.50 \%, \mathrm{~N} 4.70 \%, \mathrm{H} 4.43 \%)$ 相符合. 因此, $\mathrm{PB} / \mathrm{QL}$, $\mathrm{PB} / \mathrm{AD}$ 反应的最终产物应该分别为聚溴化炔丙基喹啉 [poly(propargyl quinolinium bromide), PPQB]、聚溴化炔 丙基呋啶 [poly(propargyl acridinium bromide), PPAB] (Scheme 1). 而以下的实验事实也从另一角度证明了这 一结论: (1)我们已经证明在相同的反应条件下, PB 之间 不会发生均聚反应 ${ }^{[36]}$ (排除了第 1 种可能结构); (2)溴代 烃 PB 可以迅速和吡啶及其衍生物反应，形成相应的季 铵盐 ${ }^{[37,38]}$ (即上述两个反应体系中应分别快速形成 PQB 
和 PAB), 为 PPQB 和 PPAB 的形成提供可能; (3)通过调 节反应物中 $\mathrm{PB} / \mathrm{QL}$ (或 $\mathrm{PB} / \mathrm{AD}$ )的投料比(由 $1: 1$ 变为 $2: 1,3: 1)$, 制备了一系列的聚合产物, 对这些产物纯 化后进行 EA 测试分析, 结果显示反应产物中各元素的 含量并未发生变化, 这说明 $\mathrm{PB}$ 与 $\mathrm{PQB}$ 或 $\mathrm{PAB}$ 之间不 会发生共聚(排除了第 2 种可能结构). 上述实验事实还 说明: PPQB 和 PPAB 是分别由 PQB 和 PAB 均聚获得(如 Scheme 2 所示).

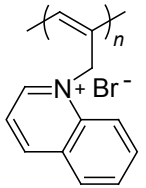

PPQB

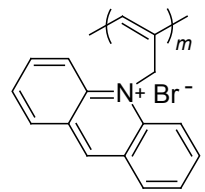

PPAB
图式 $1 \mathrm{PPQB}$ 与 PPAB 的化学结构式

Scheme 1 Chemical structures of PPQB and PPAB
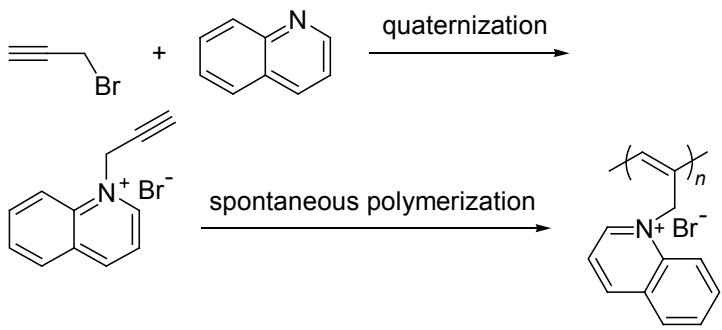

图式 2(a) $\mathrm{QL}$ 与 $\mathrm{PB}$ 反应生成 $\mathrm{PQB}$, 及 $\mathrm{PQB}$ 自聚合生成 $\mathrm{PPQB}$

Scheme 2(a) Reaction of QL and $\mathrm{PB}$ into $\mathrm{PQB}$, and polymerization of the latter into PPQB
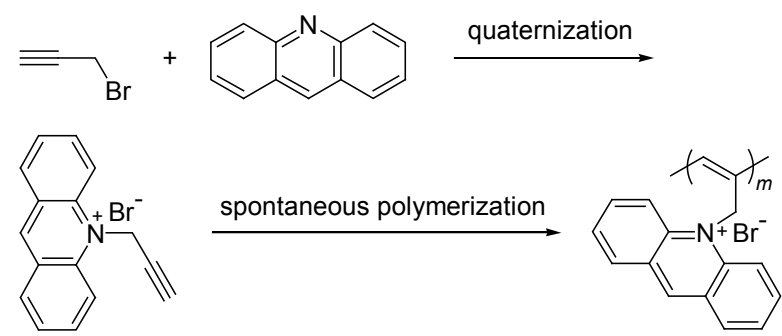

图式 2(b) $\mathrm{AD}$ 与 $\mathrm{PB}$ 反应生成 $\mathrm{PAB}$, 及 $\mathrm{PAB}$ 自聚合生成 $\mathrm{PPAB}$

Scheme 2(b) Reaction of $\mathrm{AD}$ and $\mathrm{PB}$ into $\mathrm{PAB}$, and polymerization of the latter into PPAB

在已有的工作中, 我们提出并验证了 $\mathrm{PB} / \mathrm{PD}$ 反应生 成 PPPB 的反应机理 ${ }^{[36]}$. 作为吡啶衍生物的 QL 与 $\mathrm{AD}$, 其分子结构中均包含有吡啶环(这两种分子结构中的其 它部分则为化学惰性结构), 因此 $\mathrm{PB} / \mathrm{PD}$ 反应机理应该 适用于 $\mathrm{PB} / \mathrm{QL}, \mathrm{PB} / \mathrm{AD}$ 反应: $\mathrm{PB}$ 与 $\mathrm{QL}$ (或 $\mathrm{AD}$ )之间的季 铵化反应, 不仅导致季铵盐的生成, 还使得季铵盐中的 炔基基团被活化(S3, Supporting Information); 未反应的 $\mathrm{QL}$ (或 $\mathrm{AD}$ ) 分子中氮原子的孤对电子亲核进攻季铵盐 的炔基产生碳负离子, 碳负离子继续进攻其他季铵盐的 炔基基团进行链增长反应; 如果链末端的碳负离子进攻
未反应的 $\mathrm{PB}$ 分子中的 $\mathrm{C}-\mathrm{Br}$ 键，则会导致链增长反应 的终止(Scheme 3).

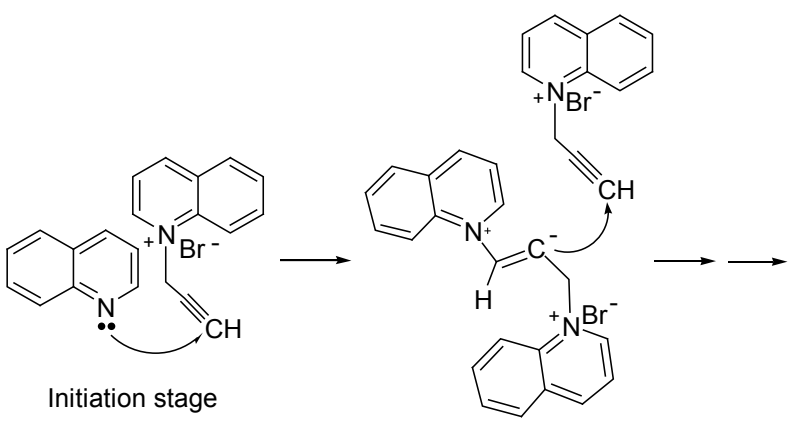

Propagation stage

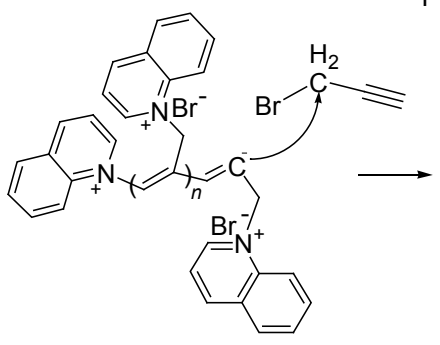

Termination stage

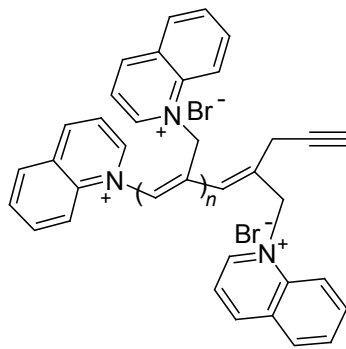

图式 3(a) $P Q B$ 聚合生成 $\mathrm{PPQB}$ 反应机理

Scheme 3(a) Mechanism of polymerization of PQB into PPQB
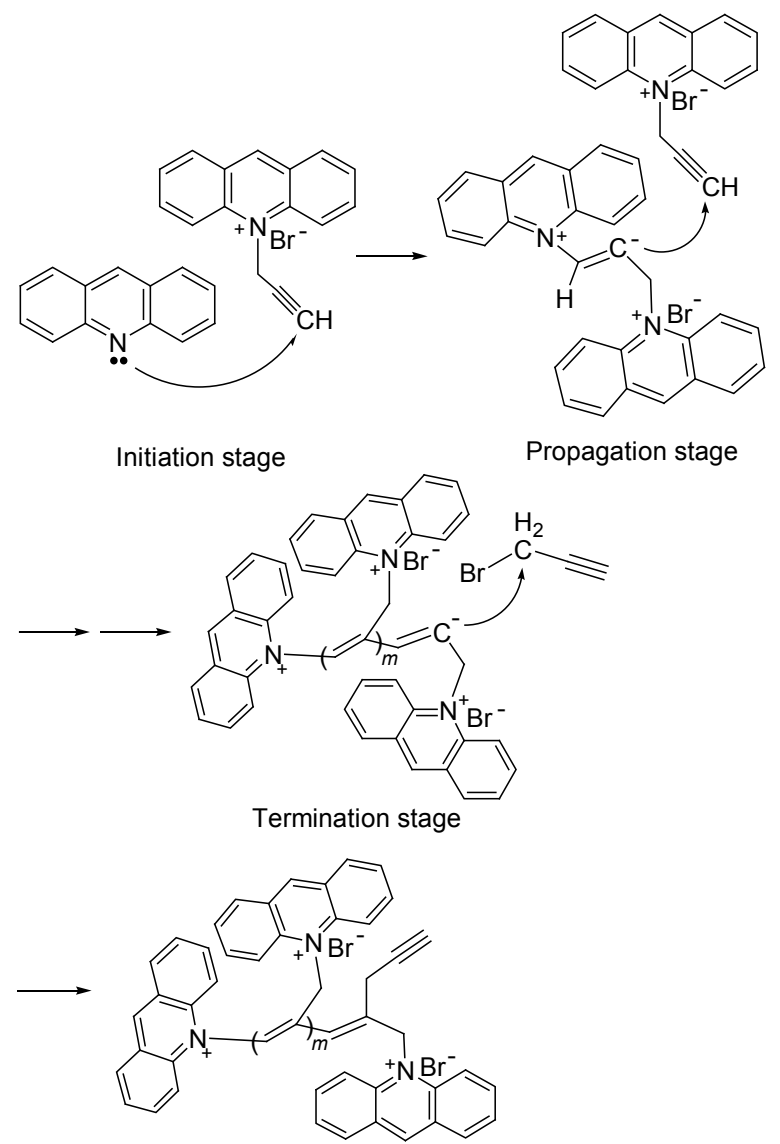

图式 3(b) PAB 聚合生成 PPAB 反应机理

Scheme 3(b) Mechanism of polymerization of PAB into PPAB 


\section{$2.2 P P Q B$ 及 $P P A B$ 的荧光性质}

PPQB, PPAB 在甲醇、乙醇、DMF、DMSO 等有机 溶剂中均具有良好的溶解性. 图 1 是 $\mathrm{PPQB}, \mathrm{PPAB}$ 的甲 醇溶液的 UV-Vis 吸收光谱. 其中, PPQB, PPAB 分别在 $318,355 \mathrm{~nm}$ 处出现最大吸收峰. 采用 $\lambda_{\mathrm{ex}}=318 \mathrm{~nm}$ 的激 发光分别激发 $\mathrm{QL}$ 与 $\mathrm{PPQB}$ 溶液(溶剂均为甲醇, 浓度均 为 $0.2 \mathrm{mg} / \mathrm{mL}$ ), 可以发现 $\mathrm{QL}$ 溶液的最大荧光发射峰位 于 $335 \mathrm{~nm}$ 处, 而 PPQB 溶液的最大荧光发射峰为宽峰, 位于 $438 \sim 538 \mathrm{~nm}$ 范围内(图 2a). 在更长波长的激发光 (如 $\lambda_{\mathrm{ex}}=451 \mathrm{~nm}$ 或 $468 \mathrm{~nm}$ ) 激发下, QL 溶液已无荧光发 射, 而 PPQB 溶液依旧保持较强的苂光发射强度, 其最 大苂光发射峰分别位于 $545 \mathrm{~nm}\left(\lambda_{\mathrm{ex}}=451 \mathrm{~nm}\right), 555 \mathrm{~nm}$ $\left(\lambda_{\mathrm{ex}}=468 \mathrm{~nm}\right)$ 处(图 2b).

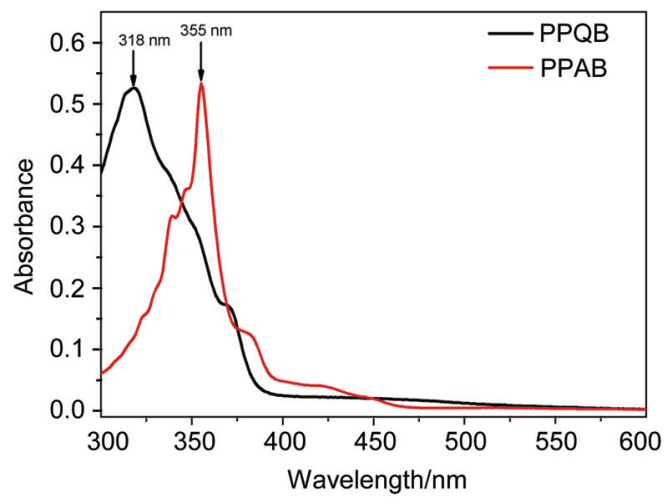

图 $1 \mathrm{PPQB}$ 与 PPAB 溶液(溶剂均为甲醇, 浓度分别为 $0.02 \mathrm{mg} / \mathrm{mL}$, $0.01 \mathrm{mg} / \mathrm{mL}$ )的 UV-Vis 吸收光谱图

Figure 1 UV-Vis absorption spectra of PPQB in methanol $(0.02$ $\mathrm{mg} / \mathrm{mL})$ and PPAB in methanol $(0.01 \mathrm{mg} / \mathrm{mL})$

对 $\mathrm{AD}$ 与 PPAB 的甲醇溶液的荧光性质进行测试, 也可以观测到类似的现象. 采用 PPAB 溶液的最大吸收 峰波长 $(355 \mathrm{~nm})$ 的激发光分别对 $\mathrm{AD}$ 溶液 $(0.2 \mathrm{mg} / \mathrm{mL})$ 与 PPAB 溶液 $(0.2 \mathrm{mg} / \mathrm{mL})$ 进行激发, PPAB 溶液的苂光强 度要明显大于 $\mathrm{AD}$ 溶液的荧光强度, 且 PPAB 溶液的最 大苂光发射峰 $\left[\lambda_{(\mathrm{em}) \max }=497 \mathrm{~nm}\right]$ 相较于 $\mathrm{AD}$ 溶液 $\left[\lambda_{(\mathrm{em}) \max }\right.$ $=417 \mathrm{~nm}$ ] 呈现出明显的红移(图 3a). 若采用更长波长 的激发光(如 $\lambda_{\mathrm{ex}}=460 \mathrm{~nm}$ )进行激发, PPAB 溶液依然发 射出较强的苂光, 其最大荧光发射峰位于 $485 \sim 530 \mathrm{~nm}$ 范围内, $\mathrm{AD}$ 溶液却无明显的荧光发射(图 3b).

\section{$2.3 \mathrm{PPQB}$ 及 $\mathrm{PPAB}$ 的导电性能}

上已述及, $\mathrm{PB}$ 与 $\mathrm{PD}$ 反应生成的 $\mathrm{PPPB}$ 在空气中具 有很强的吸水性, 对其在空气中的稳定性造成影响, 从 而限制了 PPPB 的进一步应用; 而 PPQB, PPAB 等在空 气中的吸水性比较弱(S4, Supporting Information). 原因 是相对于 PPPB 侧链的 $\mathrm{C}_{5} \mathrm{H}_{5} \mathrm{~N}^{+}$基团, PPQB, PPAB 侧链 的 $\mathrm{C}_{9} \mathrm{H}_{7} \mathrm{~N}^{+}, \mathrm{C}_{13} \mathrm{H}_{9} \mathrm{~N}^{+}$基团极性较弱, 使得聚合物的亲水 和吸水性能较 PPPB 呈显著下降, 在空气中的稳定性增 强. 因此可以分别制备出在空气中稳定的 PPQB 和
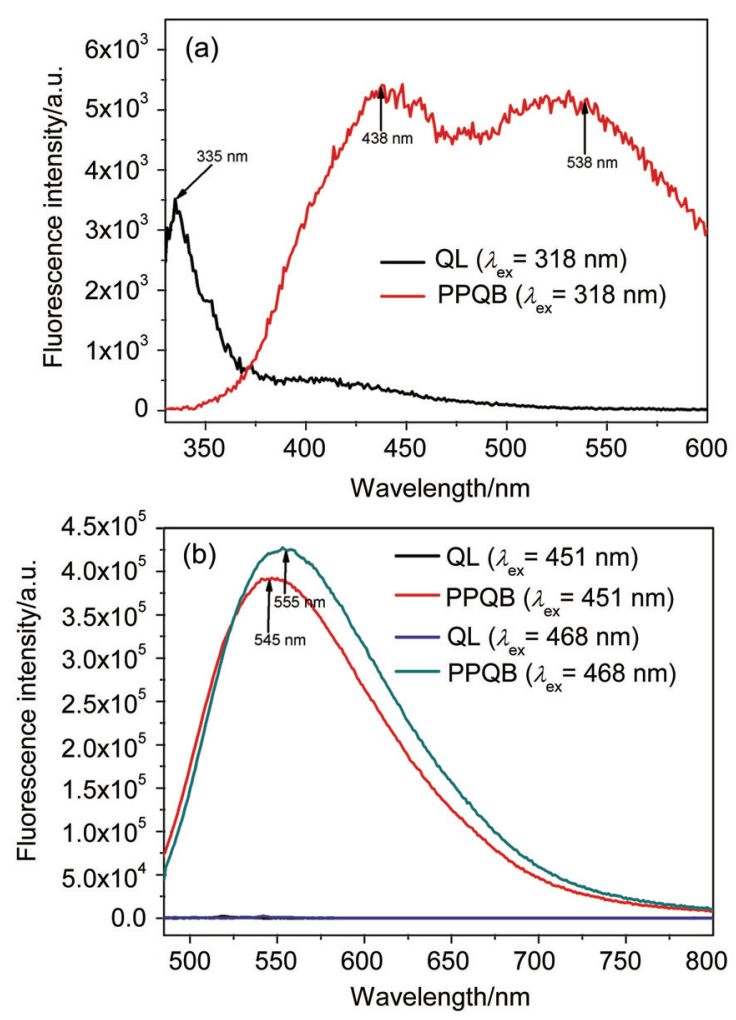

图 $2 \mathrm{QL}$ 与 $\mathrm{PPQB}$ 溶液 (溶剂均为甲醇, 浓度均为 $0.2 \mathrm{mg} / \mathrm{mL}$ ) 分别在 $\lambda_{\mathrm{ex}}=318 \mathrm{~nm}$ (a) 和 $\lambda_{\mathrm{ex}}=451 / 468 \mathrm{~nm}$ (b) 的激发光激发下的荧光发射光 谱图

Figure 2 Fluorescence emission spectra of QL and PPQB in methanol excited by lights with $\lambda_{\mathrm{ex}}=318 \mathrm{~nm}$ (a) and $\lambda_{\mathrm{ex}}=451 / 468 \mathrm{~nm}$ (b) (both concentrations of QL and PPQB solution are $0.2 \mathrm{mg} / \mathrm{mL}$ )

PPAB 薄膜并测量其导电性能. 测试结果如图 4 所示, 随着施加于薄膜器件两端的电压值的增大, 通过器件的 电流亦随之线性上升，根据图 4 可以计算出实验中所制 备的 PPQB 与 $\mathrm{PPAB}$ 薄膜器件的电阻分别为 12.1 和 21.7 $\Omega$, 表明所制备 PPQB, PPAB 薄膜具有导电性能(由于影 响该结果的因素较多, 暂未对这两种聚合物的导电性进 行更加详细的评估). 由于是在常温、干燥状态下测量的 导电性, 该导电性应该是由聚合物的共轭结构贡献的. 为证明这一观点, 我们在相同的实验条件下, 用季铵化 的聚 4-乙烯基吡定替代 PPQB 或 PPAB 测试其导电性能, 测得的电流值基本为零.

\section{3 结论}

将 $\mathrm{PB}$ 分别与 $\mathrm{QL}, \mathrm{AD}$ 直接混合反应，可以高效制 备高分子量的 PPQB 和 PPAB. 该方法具有无引发剂、 无催化剂、反应条件温和等特点. 该研究结果也进一步 证明了 PB 与吡定衍生物反应是一种新型的且具有普适 性的制备 PE-PAs 的方法. 制得的 PPQB, PPAB 聚合物 不仅具有较高的分子量, 还具有较强的荧光发射强度. 另外, 由于侧基的极性较低，使得 PPQB 与 PPAB 材料 的吸水性相较 PPPB 有明显降低, 从而可以制备出在空 

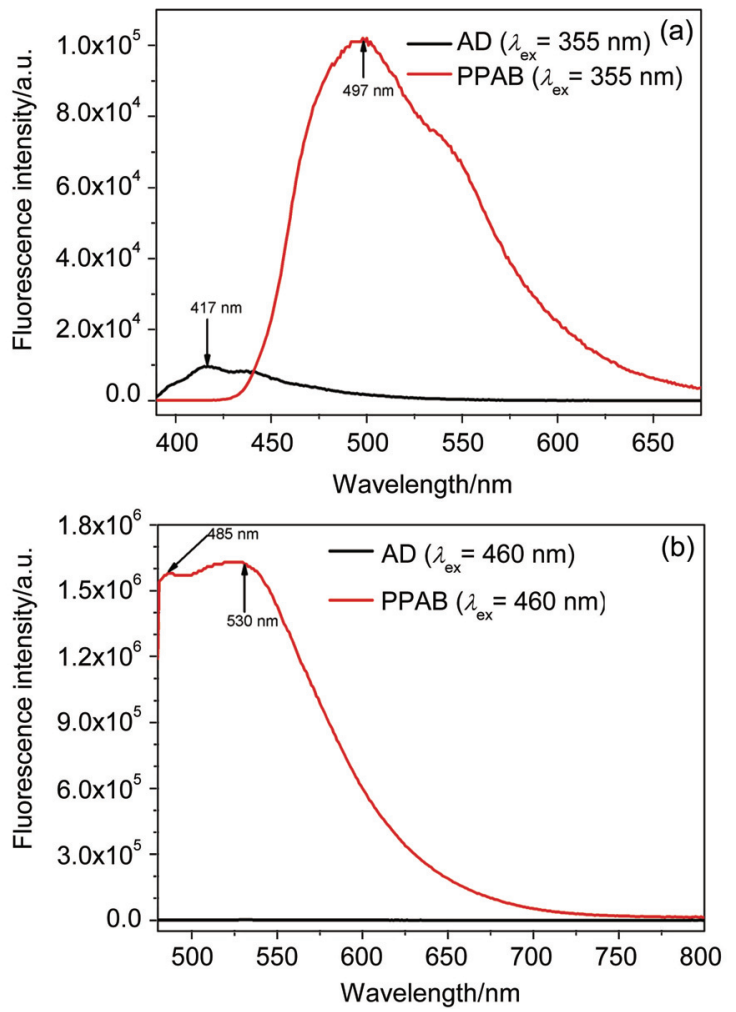

图 $3 \mathrm{AD}$ 与 $\mathrm{PPAB}$ 溶液(溶剂均为甲醇, 浓度都保持为 $0.2 \mathrm{mg} / \mathrm{mL}$ ) 分 别在 $\lambda_{\mathrm{ex}}=355 \mathrm{~nm}$ (a) 和 $\lambda_{\mathrm{ex}}=460 \mathrm{~nm}$ (b) 的激发光激发下的苂光发射光 谱图

Figure 3 Fluorescence emission spectra of $\mathrm{AD}$ and $\mathrm{PPAB}$ in methanol excited by lights with $\lambda_{\mathrm{ex}}=355 \mathrm{~nm}$ (a) and $\lambda_{\mathrm{ex}}=460 \mathrm{~nm}$ (b) (both concentrations of $\mathrm{AD}$ and $\mathrm{PPAB}$ solution are $0.2 \mathrm{mg} / \mathrm{mL}$ )

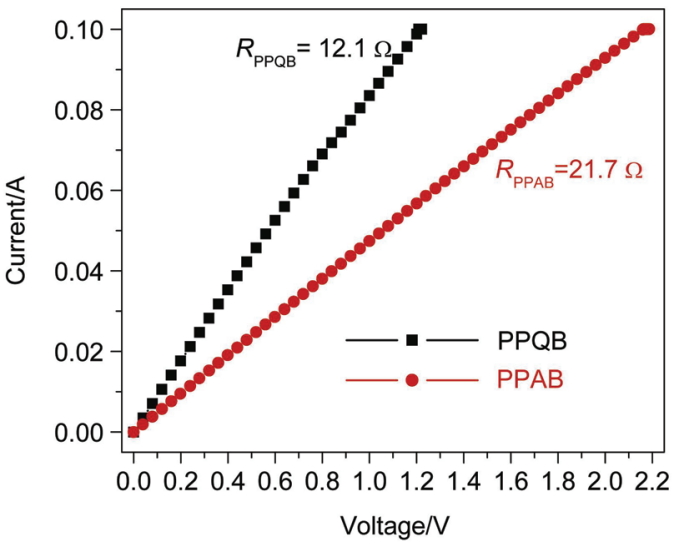

图 $4 \mathrm{PPQB}$ 与 $\mathrm{PPAB}$ 薄膜的 $I-V$ 曲线图

Figure $4 \quad I-V$ curves of PPQB and PPAB films

气中稳定的块体和薄膜材料. 对薄膜导电性的测试表 明, PPQB 和 PPAB 薄膜均具有较好的导电性能.

\section{4 实验部分}

\subsection{PPQB 的制备}

向 $20 \mathrm{~mL}$ 洁净干燥的 Schlenk 瓶中依次加入 2.36 $\mathrm{mL}(0.02 \mathrm{~mol}) \mathrm{QL}$ 和 $2 \mathrm{~mL}$ DMF，室温条件下混合均匀，
继续向 Schlenk 瓶中加入 $1.5 \mathrm{~mL}(0.02 \mathrm{~mol}) \mathrm{PB}$, 于室温 条件下摚拌 $10 \mathrm{~min}$. 然后将反应液经过 “冻冷一脱气一 充气一熔融”循环处理 3 次以除水除氧, 在 $\mathrm{Ar}$ 气保护下 于 $60{ }^{\circ} \mathrm{C}$ 油浴中反应 $100 \mathrm{~h}$ 后, 再置于液氮中冷冻终止 反应. 反应产物经甲醇溶解后，滴入到 10 倍体积的乙醚 中沉淀得到固体产物. 反复进行上述 “溶解一沉淀一再 溶解” 过程共 3 次, 获得红褐色固体产物. 将固体产物 于室温条件下真空干燥 $24 \mathrm{~h}$ 后, 再置入干燥器中避光保 存.

\subsection{PPAB 的制备}

向 $20 \mathrm{~mL}$ 洁净干燥的 Schlenk 瓶中依次加入 $3.58 \mathrm{~g}$ $(0.02 \mathrm{~mol}) \mathrm{AD}$ 和 $3 \mathrm{~mL} \mathrm{DMF}$ ，室温条件下混合均匀，继 续向 Schlenk 瓶中加入 $1.5 \mathrm{~mL}(0.02 \mathrm{~mol}) \mathrm{PB}$, 于室温条 件下搅拌 $10 \mathrm{~min}$. 后续操作同 PPQB 的制备过程. 最终 获得的 PPAB 为浅黄色固体, 置入干燥器中避光保存.

\section{References}

[1] Jiang, H.; Taranekar, P.; Reynolds, J. R.; Schanze, K. S. Angew. Chem. Int. Ed. 2009, 48, 4300.

[2] Ye, H. Y.; Li, W.; Li, W. S. Chin. J. Org. Chem. 2012, 32, 266. (叶 怀英, 李文, 李维实, 有机化学, 2012, 32, 266.)

[3] Huo, Y. P.; Zeng, H. P.; Jiang, H. F. Chin. J. Org. Chem. 2004, 24 1191. (霍延平, 曾和平, 江焕峰, 有机化学, 2004, 24, 1191.)

[4] Chen, L.; Shen, X. X.; Chen, Y. W. Chin. J. Chem. 2012, 30, 2219.

[5] Zeng, W. N.; Qi, J. P.; Wang, L. Y.; Cao, D. R. Chin. J. Org. Chem. 2009，29，1858. (曾文南，元金萍，汪凌云，曹德榕，有机化学， 2009, 29, 1858.)

[6] Chen, Y. G.; Xu, B. M.; He, Z. K.; Xie, W. H. Acta Chim. Sinica 2011，69，1361. (陈彦国，徐保明，何治柯，谢卫红，化学学报， 2011, 69, 1361.)

[7] Xiao, Y.; Hu, Y. Q.; Huang, H. M.; Yin, D. L.; Xiao, X. M. Acta Chim. Sinica 2011, 69, 838. (肖毅, 胡玉琴, 黄红梅, 尹笃林, 肖 小明, 化学学报, 2011, 69, 838.)

[8] Du, J.; Zhao, D.; Chen, Y. G.; He, Z. K. Acta Chim. Sinica 2006, 64 , 963. (杜鹃，赵丹，陈彦国，何治柯，化学学报, 2006, 64, 963.)

[9] Hao, D.; Sun, L. L.; Jin, Y. Q.; Zhu, C. Q. Acta Chim. Sinica 2010, 68,2215 . (郝丹, 孙礼林, 晋英琼, 朱昌青, 化学学报, 2010, 68, 2215.)

[10] Jin, Y. Q.; Sun, L. L.; Hao, D.; Yu, R.; Qian, Z. S.; Zhu, C. Q. Chin. J. Chem. 2011, 29, 575 .

[11] Ito, T.; Shirakawa, H.; Ikeda, S. J. Polym. Sci., Polym. Chem. Ed. 1974, 12,11 .

[12] Chiang, C. K.; Fincher, C. R.; Park, Y. W.; Heeger, A. J.; Shirakawa, H.; Louis, E. J.; Gau, S. C.; Macdiarmid, A. G. Phys. Rev. Lett. 1977, 39,1098

[13] Shirakawa, H.; Louis, E. J.; Macdiarmid, A. G.; Chiang, C. K.; Heeger, A. J. J. Chem. Soc., Chem. Commun. 1977, 578.

[14] Shirakawa, H. Angew. Chem. Int. Ed. 2001, 40, 2575.

[15] MacDiarmid, A. G. Angew. Chem. Int. Ed. 2001, 40, 2581

[16] Heeger, A. J. Angew. Chem. Int. Ed. 2001, 40, 2591.

[17] Choi, S. K.; Gal, Y. S.; Jin, S. H.; Kim, H. K. Chem. Rev. 2000, 100, 1645.

[18] Mayershofer, M. G.; Nuyken, O. J. Polym. Sci., Part A: Polym. Chem. 2005, 43, 5723 .

[19] Masuda, T.; Higashimura, T. Acc. Chem. Res. 1984, 17, 51.

[20] Lam, J. W. Y.; Tang, B. Z. J. Polym. Sci., Part A: Polym. Chem. 2003, 41, 2607.

[21] Lam, J. W. Y.; Tang, B. Z. Acc. Chem. Res. 2005, 38, 745.

[22] Masuda, T. J. Polym. Sci., Part A: Polym. Chem. 2007, 45, 165

[23] Li, C. H.; Li, Y. L. Macromol. Chem. Phys. 2008, 209, 1542.

[24] Liu, J. Z.; Lam, J. W. Y.; Tang, B. Z. Chem. Rev. 2009, 109, 5799.

[25] Kawasaki, M.; Masuda, T.; Higashimura, T. Polym. J. 1983, 15, 767.

[26] Gal, Y. S.; Choi, S. K. Eur. Polym. J. 1995, 31, 941.

[27] Kang, K. L.; Kim, S. H.; Cho, H. N.; Choi, K. Y.; Choi, S. K. Macromolecules $1993,26,4539$. 
[28] Kim, S. H.; Choi, S. J.; Park, J. W.; Cho, H. N.; Choi, S. K. Macromolecules 1994, 27, 2339.

[29] Choi, D. C.; Kim, S. H.; Lee, J. H.; Cho, H. N.; Choi, S. K. Macromolecules 1997, 30, 176.

[30] Kargin, V. A.; Kabanov, V. A. US 3658942, 1972 [Chem. Abstr. 1972, 74, 88376].

[31] Subramanyam, S.; Blumstein, A. Macromolecules 1991, 24, 2668.

[32] Gal, Y. S.; Lee, W. C.; Choi, S. K. Bull. Korean Chem. Soc. 1997, $18,265$.

[33] Gal, Y. S.; Lee, W. C.; Kim, S. Y.; Park, J. W.; Jin, S. H.; Koh, K. N.;
Kim, S. H. J. Polym. Sci., Part A: Polym. Chem. 2001, 39, 3151.

[34] Kwak, G.; Jin, S. H.; Park, J. W.; Gal, Y. S. Macromol. Chem. Phys. 2008, 209, 1770.

[35] Gal, Y. S.; Jin, S. H.; Park, J. W.; Lim, K. T. J. Polym. Sci., Part A: Polym. Chem. 2009, 47, 6153.

[36] Zhou, C. M.; Gao, Y.; Chen, D. Y. J. Phys. Chem. B 2012, 116 , 11552.

[37] Huang, R.; Chen, D. Y.; Jiang, M. J. Mater. Chem. 2010, 20, 9988.

[38] Chen, D. Y.; Peng, H. S.; Jiang, M. Macromolecules 2003, 36, 2576.

(Cheng, B.; Fan, Y.) 